Claudia Patricia Meneses Amaya

Ismael Estrada Cañas"

Clandia Patricia Cote Peña"

Universidad Industrial de Santander Bucaramanga, Colombia

Recibido: 1 de marzo de 2017

Concepto de evaluación: 10 de mayo de 2017

Aprobado: 23 de junio de 2017

Artículo de investigación

(C) 2017 Universidad Católica de Colombia.

Facultad de Ciencias

Económicas y Administrativas.

Todos los derechos reservados

* M.Sc. (c) en Economía y Desarrollo,

Economista. Investigadora del grupo Estudios en Microeconomía Aplicada y Regulación (EMAR), Escuela de Economía y

Administración, Universidad Industrial de Santander.

Bucaramanga, Colombia. Dirección postal: Carrera 27 - Calle 9, Ciudad Universitaria, Bucaramanga, Colombia. Correo electrónico: claudia.meneses@correo.uis.edu.co

** M.Sc. en Economía y Desarrollo, Economista. Docenteinvestigador del grupo Estudios en Microeconomía Aplicada y

Regulación (EMAR), Escuela de Economía y Administración, Universidad Industrial de Santander. Dirección postal: Carrera 27 Calle 9, Ciudad Universitaria, Bucaramanga, Colombia. Correo electrónico: ismael.estrada@correo.uis.edu.co
Finanz. polit. econ., ISSN: 2248-6046, Vol. 9, No. 2, julio-diciembre, 2017, pp. 397-417

http://dx.doi.org/10.14718/revfinanzpolitecon.2017.9.2.9

\section{Implicaciones de la inversión en recursos intangibles sobre la capacidad exportadora de las empresas de Santander, Colombia*}

\section{RESUMEN}

Este artículo analiza la incidencia de la inversión en recursos intangibles sobre la capacidad exportadora de las empresas de Santander; para ello, se estima su correlación mediante dos modelos de regresión lineal uniecuacional: uno sin corrección de sesgo y otro aplicando la corrección de sesgo propuesta por Heckman (1979). Los resultados muestran que existe una relación directa entre la inversión en patentes, know how, crédito mercantil, licencias, marcas, concesiones, franquicias y derechos, y la capacidad de penetración en el mercado exterior. Sin embargo, los tipos de recursos inmateriales en los que se realizan mayores inversiones son aquellos que tienen menor incidencia en la capacidad exportadora.

Palabras clave: capacidad exportadora, conocimiento, inversión en recursos intangibles, Santander.

JEL: F23, M15, O32, O38

\section{Implications of investment in intangible resources on the exporting capacity of companies in Santander, Colombia}

\section{ABSTRACT}

This article analyzes the impact of investments in intangible resources on the exporting capacity of companies in Santander; to this effect, their correlation is estimated using two uniequational linear regression models: one without bias correction and the other one applying the bias correction proposed by Heckman (1979). The results show that there is a direct relationship between investments in patents, know-how, merchant credit, licenses, brands, concessions, franchises and rights, and the ability to expand to foreign markets.

\footnotetext{
* Este artículo forma parte de los resultados obtenidos en el trabajo de grado titulado "Implicaciones estratégicas de los recursos intangibles en la competitividad empresarial: un análisis empírico del caso santandereano", el cual fue presentado en 2014 en la Universidad Industrial de Santander.
} 
*** Especialista en Alta Gerencia y en Dirección de Empresas, Economista.

Docente-investigadora del grupo

Estudios en Microeconomía Aplicada y Regulación (EMAR),

Escuela de Economía y

Administración, Universidad

Industrial de Santander. Dirección

postal: Carrera 27 - Calle 9, Ciudad

Universitaria, Bucaramanga,

Colombia. Correo electrónico:

ccote@uis.edu.co
Nevertheless, the types of intangible resources that receive the largest investments are those that have the least impact on export capacity.

Keywords: export capacity, knowledge, investment in intangible resources, Santander.

\section{Implicações do investimento em recursos intangíveis sobre a capacidade exportadora das empresas de Santander, Colômbia}

\section{RESUMO}

Este artigo analisa a incidência do investimento em recursos intangíveis sobre a capacidade exportadora das empresas de Santander; para isso, é estimada a sua correlação mediante dois modelos de regressão linear uniequacional: um sem correção de viés e outro aplicando a correção de viés proposta por Heckman (1979). Os resultados mostram que existe uma relação direta entre o investimento em patentes, know how, crédito mercantil, licenças, marcas, concessões, franquias e direitos e a capacidade de inserção no mercado exterior. Porém, os tipos de recursos imateriais nos quais são realizados maiores investimentos são os que têm menor incidência na capacidade exportadora.

Palavras-chave: capacidade exportadora, conhecimento, investimento em recursos intangíveis, Santander. 


\section{INTRODUCCIÓN}

El acelerado proceso de globalización y el avance científico y tecnológico de las últimas décadas han incrementado exponencialmente la productividad total de los factores y le han permitido a la empresa moderna alcanzar economías de escala. Este hecho ha consolidado la denominada sociedad basada en el conocimiento, en la que el conocimiento se ha convertido en un recurso estratégico para dinamizar el crecimiento empresarial y potenciar el desarrollo económico. En este contexto se enmarca la economía basada en el conocimiento y el aprendizaje (EBCA), la cual, de acuerdo con Peluffo y Catalán (2002), se fundamenta en la capacidad de innovar y crear valor rápidamente mediante la gestión y el aprendizaje de los conocimientos tácitos y explícitos de la organización.

En este marco, la inversión en recursos intangibles ha cobrado relevancia estratégica debido a que fortalece el capital intelectual o stock de conocimiento organizacional $y$, por ende, permite obtener resultados empresariales eficientes. Cabe anotar que según la teoría de los recursos y capacidades (Wernerfelt, 1984; Barney, 1991) y el modelo de dirección estratégica por competencias (Bueno, 1998), es precisamente esta eficiencia la que otorga competitividad estratégica en el mercado internacional; no obstante, para el caso del departamento de Santander no existe evidencia del impacto que tienen dichos recursos en la capacidad exportadora de las empresas.

De acuerdo con lo anterior, en este artículo se analiza la incidencia que tiene la inversión en recursos intangibles sobre la capacidad exportadora de las empresas santandereanas que se encuentran constituidas legalmente como sociedades mercantiles; para tal fin, se estiman dos modelos de regresión lineal uniecuacional por el método de mínimos cuadrados ordinarios (MCO): el primero sin corrección de sesgo y el segundo aplicando la corrección de sesgo propuesta por Heckman (1979).

En ambas regresiones se toman los flujos de exportación ponderados por el total de ventas de las empresas como variable dependiente proxy de su capacidad exportadora; por otro lado, como variables explicativas se contemplan los distintos tipos de inversión en activos intangibles que admite el Plan Único de Cuentas (Instituto Nacional de Contadores Públicos, 1993), además de algunos controles propios de las características empresariales. Para realizar el contraste, se utiliza la información contable, financiera y comercial que las sociedades mercantiles de Santander reportaron a la Superintendencia de Sociedades de Colombia ${ }^{1}$ en el 2012.

Se considera que la inversión en recursos intangibles favorece significativamente la capacidad exportadora de las empresas, debido a que consolida una ventaja competitiva sostenible que les permite posicionarse en el mercado exterior; por ello, la contribución de este estudio consiste en hacer un análisis descriptivo y correlacional que haga posible probar esta hipótesis y aportar evidencia de su impacto en el departamento de Santander. En concreto, se busca dar respuesta al siguiente interrogante: ¿cuál es la incidencia que tiene la inversión en recursos intangibles sobre la capacidad exportadora de las empresas de Santander? La importancia de este ejercicio radica en que puede servir como derrotero para encauzar las decisiones de inversión de la comunidad empresarial santandereana.

\section{FUNDAMENTACIÓN TEÓRICA}

El proceso de transformación social ha tenido como protagonistas el continuo desarrollo científico y tecnológico y la configuración de nuevos modos de producción; así, los avances de la última década del siglo XX y de lo corrido del siglo XXI han permitido que el conocimiento sea concebido como un factor

1 "Organismo técnico adscrito al Ministerio de Comercio, Industria y Turismo, con personería jurídica, autonomía administrativa y patrimonio propio, mediante el cual el Presidente de la República ejerce la inspección, vigilancia y control de las sociedades mercantiles, así como las facultades que le señala la ley en relación con otros entes, personas jurídicas y personas naturales, (Superintendencia de Sociedades de Colombia, 2017). 
fundamental en el proceso productivo, un motor dinamizador del cambio empresarial y un recurso estratégico para la consecución del desarrollo, lo que constituyó el origen de la era del conocimiento o de los intangibles (Bueno, 2013).

Lo anterior permitió la consolidación de la sociedad basada en el conocimiento (SBC), la cual se revela como "un nuevo tipo de organización social, amplificadora e innovadora, en la que cada actor puede dar lugar a nuevo conocimiento" (Marín, 2012, p. 56). En este sentido, las alteraciones en el entorno económico llevaron a que la SBC asumiera como estructura productiva y creadora de valor a la economía basada en el conocimiento y el aprendizaje (EBCA). Esta nueva dimensión de la economía "se centra en la capacidad de innovar y crear valor más rápido en base al conocimiento y a su rápida actualización en diversos ámbitos por medio del aprendizaje" (Peluffo y Catalán, 2002, p. 8).

El conocimiento se ha incorporado como un factor estratégico en los procesos organizacionales. Así, cuando una empresa pone en acción su conocimiento, este se traduce en intangibles, los cuales son fuente clave para la productividad, la innovación y la creación de valor. Desde este marco, diversos desarrollos teóricos han demostrado que el valor de una empresa se basa en el saber hacer; además, su eficiencia es fruto de los recursos inmateriales internos, como el conocimiento que apropia para dinamizar su estrategia (Villafañe, 2005). Esta nueva visión se sustenta en el vínculo conceptual que existe entre la teoría de los recursos y capacidades y el modelo de dirección estratégica por competencias; dichos enfoques complementarios sirven como fundamento teórico para analizar las implicaciones de la inversión en intangibles sobre los resultados empresariales.

\section{Teoría de los recursos y capacidades}

La teoría de los recursos y capacidades indaga sobre dos aspectos fundamentales: el primero se refiere a cómo y por qué las empresas alcanzan una ventaja competitiva y son capaces de sostenerla; el segundo alude a "los motivos por los cuales las empresas, que desarrollan su actividad en el mismo entorno competitivo y que, estarían sujetas a los mismos factores de éxito identificados en el sector económico, obtienen niveles de rentabilidad diferenciados" (Huerta, Navas y Almodóvar, 2004, p. 88).

Wernerfelt (1984) y Barney (1991), exponentes de esta teoría, centran su análisis en la empresa, y no en el sector en el que esta participa. Así, le dan un giro a la definición de empresa, al considerarla ya no como una cartera de negocios, sino como un conjunto de recursos únicos y capacidades distintivas que la diferencian de la competencia. Por otro lado, Navas (2015), citando a Guerras y Navas (2015), sostiene que "los recursos y capacidades de la empresa pueden entenderse como el conjunto de elementos, factores, activos, habilidades, atributos que la empresa posee o controla y que le permiten formular y poner en marcha una estrategia" (p. 9).

La estructura teórica del enfoque de los recursos y las capacidades se sustenta en dos hipótesis fundamentales (Barney, 1991). La primera es que "las empresas que compiten en una industria o sector son heterogéneas entre sí respecto a los recursos estratégicos que controlan" (Barrios, 2005, p. 28), es decir, las empresas difieren entre sí en la medida en que atesoran recursos de distintos tipos sobre los que pueden sustentar su ventaja competitiva. Al respecto conviene decir que "los recursos poseen diferentes niveles de eficiencia, siendo algunos superiores a otros, por lo que las empresas dotadas con recursos superiores tendrán más posibilidad de obtener mayores resultados económicos" (García-Zambrano, García-Merino y Rodríguez, 2012, p. 16). La segunda hipótesis hace hincapié en que los recursos no son perfectamente móviles entre una empresa y otra, por lo tanto, la heterogeneidad a la que se refiere la primera hipótesis puede permanecer por más tiempo (Barney, 1991; citado en Barrios, 2005). Esta movilidad imperfecta se respalda en el hecho de que los recursos y las capacidades no están disponibles para todas las empresas en las mismas condiciones.

De hecho, a partir de las premisas anteriores, las empresas tienen la posibilidad de poseer recursos con niveles de eficiencia superiores - llamados por Barney (1991) factores estratégicos y por 
Wernerfelt (1984) recursos críticos-. Barney (1991) señala que este tipo de recursos se diferencian de los demás porque son valiosos, escasos, inimitables y difíciles de sustituir. La posesión de estos factores le proporciona a cada empresa una dotación de recursos distinta, lo que les permite lograr su propia ventaja competitiva sostenible $y$, por ende, una rentabilidad diferenciada. En consecuencia, esta línea de pensamiento defiende la asimetría y heterogeneidad de los recursos entre las firmas como causa principal de la eficiencia en los resultados empresariales (Barney, 1991; Wernerfelt, 1984).

En definitiva, esta corriente de pensamiento considera los recursos internos de la empresa como el cimiento de su estrategia y, por consiguiente, como fuente del éxito organizacional. De este modo, se pone de manifiesto el potencial estratégico que poseen los recursos intangibles, puesto que, al estar basados en conocimiento e información y carecer de una estructura física, son los que pueden cumplir con mayor facilidad las características de los recursos críticos o factores estratégicos. En concreto, su valor se aumenta mediante la repetición y experimentación, no suelen estar disponibles en el mercado y su naturaleza tácita y la protección conferida por los derechos de propiedad hace difícil copiarlos y sustituirlos (García-Rodríguez, 2004). No obstante, la consecución de resultados superiores al promedio no solo está determinada por la habilidad empresarial de adquirir recursos críticos, sino también por la destreza para coordinarlos (Aragón y Rubio, 2005).

\section{Modelo de dirección estratégica por competencias}

La puesta en marcha de actividades de innovación y desarrollo por parte de las empresas ha incrementado notablemente la generación de capital intelectual, el cual contempla los flujos de conocimiento e información y, en general, todos aquellos recursos organizativos de naturaleza intangible que contribuyen a la creación de valor (Ordóñez, 2004). Este tipo de capital es ampliamente reconocido como la fuente más importante para agregar valor y lograr ventaja competitiva en las organizaciones (Bontis,
Janoševic y Dženopoljac, 2016; Beşkese, Bozbura y Aldemir, 2014; Bontis y Mention, 2013). Sin embargo, el capital intelectual requiere un direccionamiento estratégico que permita gestionarlo eficientemente; por tal motivo, un sinnúmero de investigadores ha presentado distintos enfoques sobre la manera de direccionar una organización.

Bueno y Morcillo (1997) realizaron un primer acercamiento a través del postulado dirección por competencias básicas distintivas (DCBD), que se presenta como "un paradigma de la dirección estratégica que busca identificar la fuente real de ventaja competitiva sostenida bajo un enfoque de eficiencia" (Uribe, 2001, p. 28). En este marco, el éxito de la organización es atribuido a la existencia de competencias básicas distintivas (FernándezJardón y Martos, 2014), las cuales comprenden conocimiento tácito y explícito, habilidades, destrezas y experiencia; es decir, se sustentan en recursos intangibles. Además, son las que le permiten a la empresa llevar a cabo el proceso de administración estratégica e incrementar su valor agregado.

Las competencias básicas distintivas se pueden dividir en competencias tecnológicas, organizativas, relacionales y personales. Henao y Londoño (2012) las definen de la siguiente manera:

a) Competencias tecnológicas. Son las capacidades de diseñar y fabricar que tiene la empresa, es decir, el stock de tecnologías, el saber cómo aplicarlas y su capacidad de innovación.

b) Competencias organizativas. Se asocian a la coordinación de las capacidades, los recursos y las rutinas a través de la estructura organizativa de la empresa; están conformadas por el diseño de las tareas, las rutinas, los métodos, las estructuras de poder y los sistemas de dirección e información.

c) Competencias relacionales. Se refieren a procesos orientados hacia los diferentes grupos de interés de la organización a fin de consolidar relaciones externas favorables. Algunos ejemplos son la imagen, el prestigio, la lealtad del cliente, los enlaces 
con proveedores, la capacidad de negociación con entidades financieras y el poder comercial.

d) Competencias personales. Son el conjunto de capacidades tácitas o explícitas de las personas, es el ethos de la organización, que está compuesto por aptitudes o conocimientos (lo que saben hacer), habilidades (lo que pueden hacer) y actitudes o comportamientos (lo que quieren hacer).

Desde esta perspectiva, Bueno (1998) enriquece el enfoque de la DCBD al formular el modelo de dirección estratégica por competencias, el cual profundiza en la gestión del capital intelectual o stock de recursos intangibles. Este modelo especifica que el fundamento de la ventaja competitiva es la competencia esencial, la cual se obtiene combinando las competencias básicas distintivas. De este modo, se puede precisar que si bien las competencias distintivas son insumos elementales para el direccionamiento estratégico, es la competencia esencial la que realmente permite que una organización se diferencie de las demás y que su presencia en el mercado se consolide de manera competitiva. Esta aseveración se sustenta en que las competencias básicas por sí solas no proporcionan resultados eficientes, sino que su gestión es la que permite obtener el éxito.

Bueno (1998) define el capital intelectual de una empresa como "un conjunto de competencias básicas distintivas de carácter intangible que permiten crear y sostener la ventaja competitiva" (p. 221), lo que sugiere que la competencia esencial de una empresa es su capital intelectual. Así, esta competencia está conformada por tres habilidades distintivas: una de origen estructural (capital tecnológico y organizativo), otra del ámbito o entorno socioeconómico (capital relacional) y la última de carácter personal (capital humano) (Fuentes, Osorio y Mungaray, 2016).. Lo anterior se ilustra en el figura 1.

En consecuencia, este modelo cobra relevancia por cuanto contribuye a la gestión de los recursos intangibles, es decir, los consolida como capital intelectual; de esta forma, cuando una empresa - a través de la gestión de sus recursos intangibles - implementa una estrategia que le permite fortalecer su capacidad productiva, incrementar su participación en el mercado, internacionalizarse y obtener un desempeño superior al de sus competidores, generalmente alcanza el éxito organizacional.

\section{DATOS Y ESTADÍSTICAS DESCRIPTIVAS}

\section{Datos de estudio}

La base de datos empleada corresponde a los estados contables y financieros del 2012 reportados al Sistema de Información y Reporte Empresarial (SIREM) por parte de las empresas de Santander sujetas a inspección, control y vigilancia de la

Figura 1.

Modelo de dirección estratégica por competencias

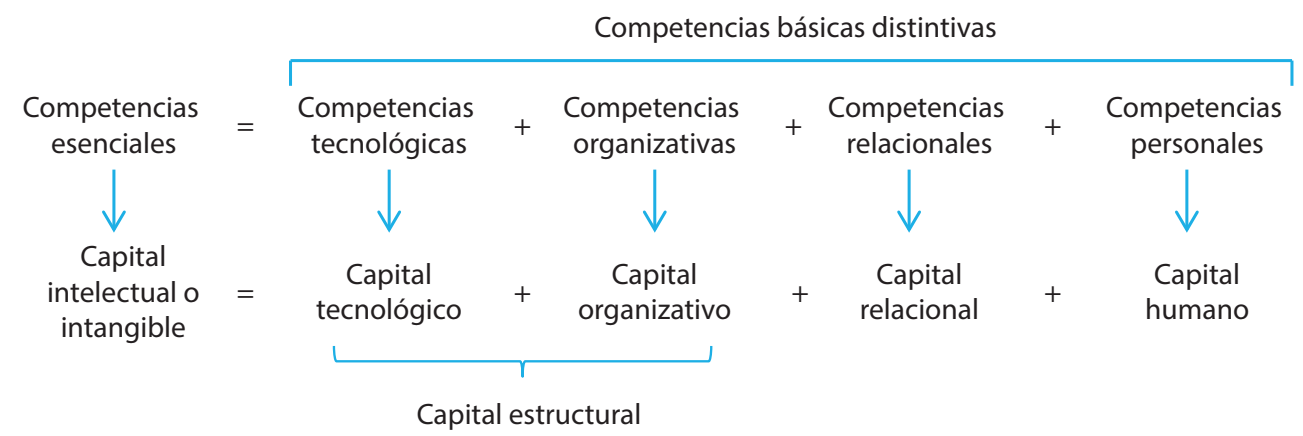

Fuente: elaboración de los autores con base en Bueno (1998). 
Superintendencia de Sociedades de Colombia. El conjunto de datos considerado en este estudio incluye información contable, financiera y económica de una muestra representativa de 797 empresas del sector real que forman parte del tejido empresarial del departamento de Santander.

EI SIREM es el medio por el cual la Superintendencia de Sociedades de Colombia hace públicos los estados financieros que suministran las empresas sometidas a su vigilancia (Decreto 4350 de 2006), control (artículo 85 de la Ley 222 de 1995) e inspección (artículo 83 de la Ley 222 de 1995). Los datos proporcionados por el SIREM se presentan de forma anonimizada; por ello, a partir de los resultados de esta investigación no es posible identificar individualmente las empresas a las que corresponde la información.

Es preciso señalar que la clasificación de los recursos intangibles contemplada en los estados financieros del SIREM corresponde a lo establecido por el Instituto Nacional de Contadores Públicos de Colombia (1993), a saber: patentes, marcas, know how, licencias, derechos, concesiones y franquicias, y crédito mercantil.

\section{Características de las empresas santandereanas que invierten en intangibles}

De acuerdo con los datos de la Superintendencia de Sociedades de Colombia, el 34,5\% de las empresas santandereanas hizo algún tipo de inversión en recursos intangibles en el 2012; no obstante, en Santander no existe un consenso acerca de los rasgos distintivos de estas organizaciones. Por tal motivo, a continuación se presentan las características específicas de las empresas que invierten en intangibles en el departamento.

A partir de la información contenida en la tabla 1, es posible establecer que las empresas que invierten en intangibles tienen mayor presencia en las provincias del norte y occidente (Soto y Mares), donde se ubican los principales municipios del departamento (Girón, Barrancabermeja, Bucaramanga, Piedecuesta, Floridablanca, entre otros). Asimismo, indica que los municipios que registran menores niveles de inversión en inmateriales son precisamente los que se encuentran más alejados de la capital santandereana; de hecho, Socorro (provincia Comunera), Cimitarra (provincia de Vélez), San Gil y Aratoca (provincia de Guanentá), al estar rezagados hacia los límites del departamento, son los municipios que presentan los menores porcentajes de empresas inversoras en intangibles.

Tabla 1.

Participación de las empresas de Santander que invierten en recursos intangibles, según ubicación, 2012

\begin{tabular}{|c|c|c|}
\hline Municipio & Invierten (\%) & No invierten (\%) \\
\hline Girón & 52,6 & 47,4 \\
\hline Barrancabermeja & 35,7 & 64,3 \\
\hline Bucaramanga & 34,4 & 65,6 \\
\hline Piedecuesta & 33,3 & 66,7 \\
\hline Floridablanca & 26,1 & 73,9 \\
\hline Socorro & 20,0 & 80,0 \\
\hline San Gil & 20,0 & 80,0 \\
\hline Aratoca & 16,7 & 83,3 \\
\hline Cimitarra & 14,3 & 85,7 \\
\hline
\end{tabular}

Fuente: elaboración de los autores con base en la Superintendencia de Sociedades de Colombia (2012).

Por otra parte, cabe anotar que en Santander el $50,7 \%$ de las empresas de gran tamaño y el $42,51 \%$ de las de tamaño mediano realizan inversiones en intangibles; en contraste, para las empresas pequeñas y las catalogadas como microempresas, esta tasa solo llega al $24,25 \%$ y $22,22 \%$, respectivamente (gráfico 1). Estas cifras indican que, en promedio, las empresas grandes invierten más que las medianas, las medianas más que las pequeñas y las pequeñas más que las microempresas; todo lo cual sugiere que en Santander, tal como afirma la teoría (Gumbau, 1997; Galende y Suárez, 1998; Vargas, 2003), existe una relación directa entre el tamaño de las empresas y las decisiones de inversión en recursos intangibles.

Con respecto a la participación sectorial, se puede afirmar que las características del sector minero y de la industria manufacturera influyen de manera significativa en las decisiones de 
Gráfico 1 .

Participación de las empresas de Santander que invierten en recursos intangibles según tamaño, 2012

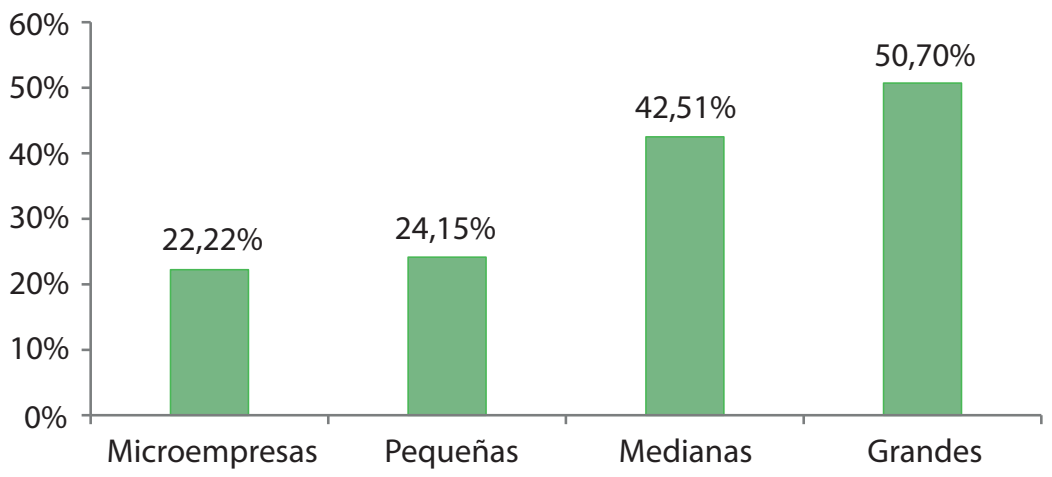

Fuente: elaboración de los autores con base en la Superintendencia de Sociedades de Colombia (2012).

Gráfico 2.

Participación sectorial de las empresas de Santander que invierten en recursos intangibles, 2012

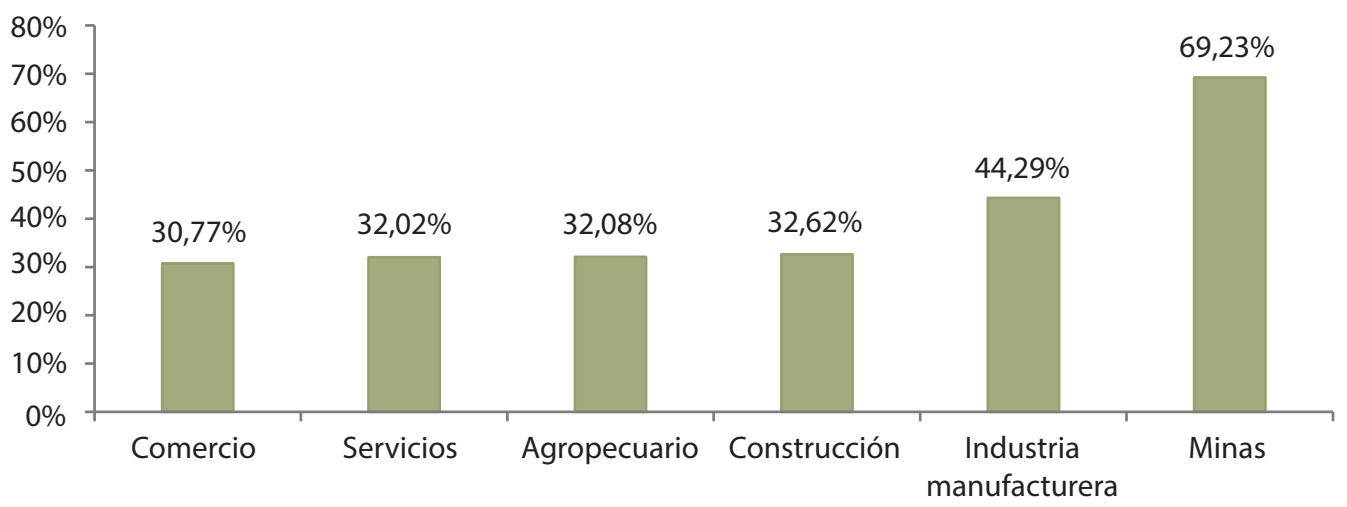

Fuente: elaboración de los autores con base en la Superintendencia de Sociedades de Colombia (2012).

inversión en inmateriales (gráfico 2). Precisamente, aspectos que sobresalen en Santander - como la tecnificación de la minería a gran escala y la transformación de materias primas en productos manufacturados- justifican que el $69,23 \%$ de las empresas mineras y el $44,29 \%$ de las industriales inviertan en recursos intangibles. Por su parte, la tasa de inversión en intangibles de los sectores de comercio, servicios, agropecuario y construcción apenas supera el $30 \%$ en cada caso. Cabe resaltar que la dinámica inversora de los sectores del departamento es consistente con la evidencia de estudios como los de Barrios (2005) y la Fundación
COTEC para la Innovación Tecnológica (2012), pero se contrapone a lo planteado por Vickery (1999).

En relación con factores como el endeudamiento empresarial (para el cual se toma la razón de endeudamiento ${ }^{2}$ ), el gráfico 3 muestra que en Santander las empresas que registran niveles de deuda más altos tienen una mayor orientación hacia el desarrollo de bienes inmateriales, mientras que aquellas con pequeñas razones de endeudamiento siguen el patrón contrario. Este hecho implica que existe una relación positiva creciente pero

2 Esta razón financiera mide la proporción del total de activos aportados por los acreedores de la empresa y se determina dividiendo el pasivo total entre el activo total (Gerencie. com, s. f.). 
Relación entre endeudamiento e inversión en intangibles en Santander, 2012

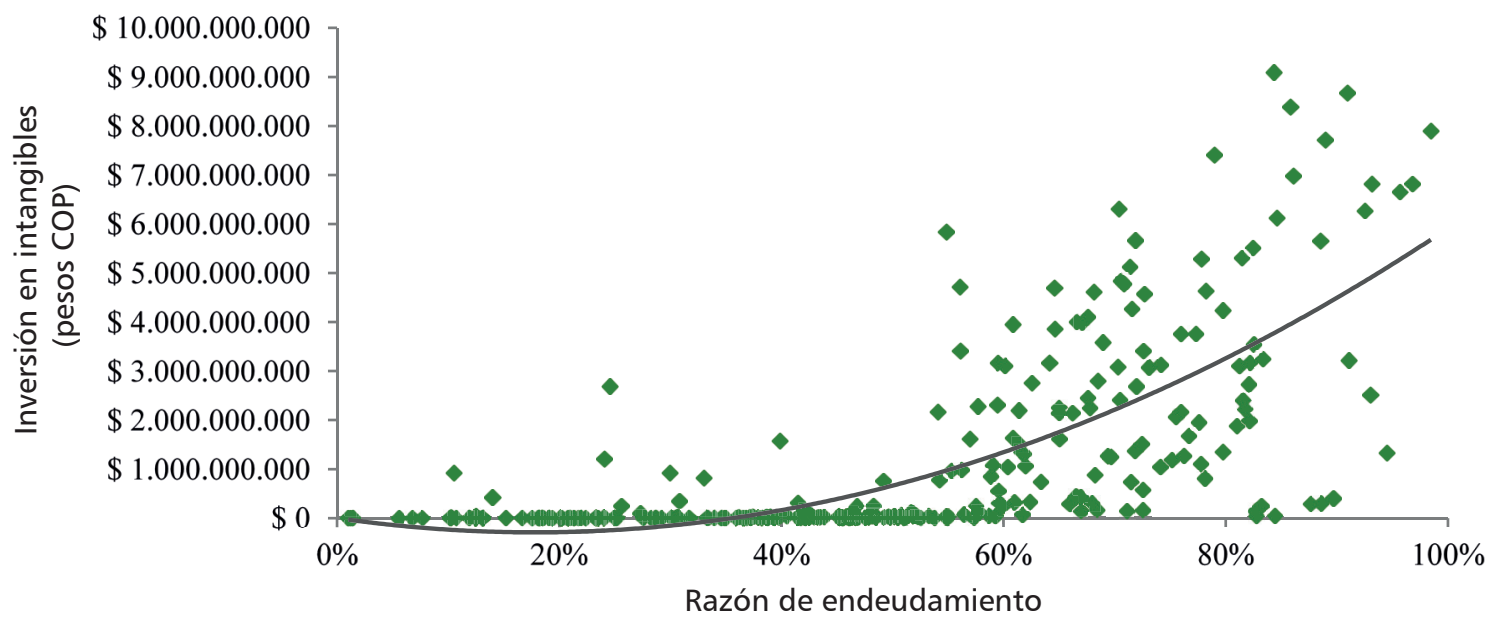

Fuente: elaboración de los autores con base en Superintendencia de Sociedades de Colombia (2012).

no causal entre el endeudamiento y el esfuerzo de inversión en recursos de naturaleza inmaterial. La estimación del coeficiente de correlación de Spearman $(\rho)$ indica que la intensidad de esta asociación es de 0,82 , con una significancia estadística del $95 \%{ }^{3}$; es decir, se confirma que existe una fuerte relación positiva entre endeudamiento e inversión en intangibles de las empresas de Santander. Estos resultados se corresponden con lo planteado por Ostad (1997) y Catasús y Gröjer (2003).

En este orden de ideas, para el caso de Santander, se evidencia una relación directa entre la inversión en intangibles y el retorno operacional sobre los activos ${ }^{4}$ (gráfico 4). No obstante, se advierte que esta asociación es marginalmente decreciente y que no tiene implicaciones de causa-efecto; en otras palabras, las empresas que realizan un mayor esfuerzo de inversión en recursos inmateriales obtienen niveles de rentabilidad más elevados que aquellas que realizan un esfuerzo bajo. Sin embargo, existe un umbral de inversión (alrededor de los 5000 millones de pesos (OP) a partir del cual la

3 Los detalles de cálculo del coeficiente de correlación de Spearman pueden ser consultados en el anexo 1.

4 Este índice mide la rentabilidad de una empresa con respecto a sus activos y se estima como la ratio entre la utilidad operacional y el total de activos que posee la organización. relación se invierte. Adicionalmente, el coeficiente de correlación de Spearman estimado en este caso es de 0,52 , con un nivel de significancia del 95\%; estos hallazgos respaldan los argumentos de Capon, Farley y Hoenig (1990), Chauvin y Hirschey (1993) y Lev y Sougiannis (1996) a favor de la existencia de una correlación positiva entre inversión en intangibles y rentabilidad.

En síntesis, las empresas santandereanas con manifiesta orientación hacia la inversión en intangibles se caracterizan por ubicarse en municipios con elevado grado de desarrollo, pertenecer principalmente a los sectores de minas e industria manufacturera y clasificarse primordialmente como grandes y medianas empresas; asimismo, factores como el endeudamiento y la rentabilidad tienen una fuerte relación positiva con las decisiones de inversión inmaterial de las organizaciones del departamento.

\section{ASPECTOS METODOLÓGICOS}

Con el fin de establecer la relación entre inversión en recursos intangibles y capacidad exportadora de las empresas de Santander, se estima un modelo de regresión lineal uniecuacional (MRLU) 
Relación entre inversión en intangibles y rentabilidad en Santander, 2012

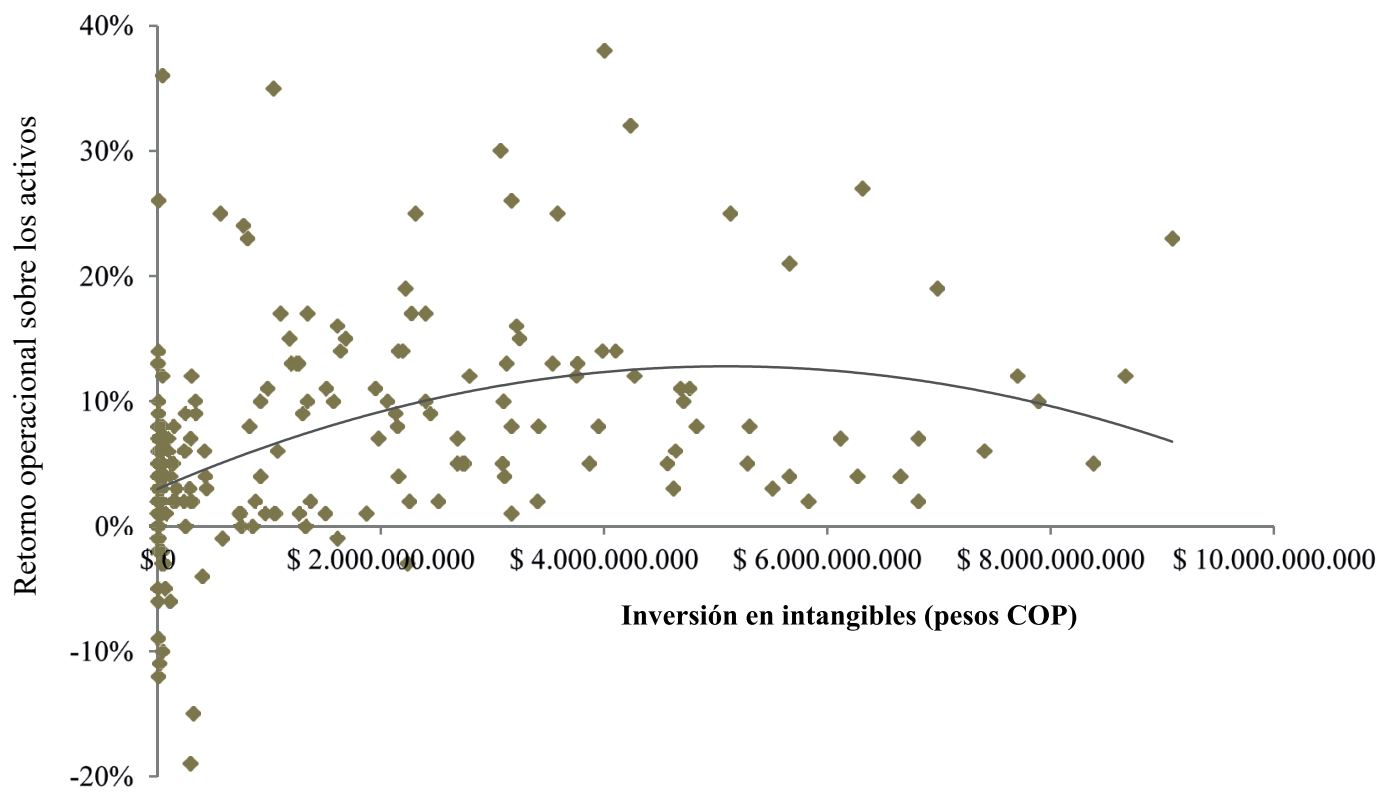

Fuente: elaboración de los autores con base en Superintendencia de Sociedades de Colombia (2012).

por el método de mínimos cuadrados ordinarios (MCO). Esta metodología permite medir el efecto de la inversión en inmateriales sobre la media de la distribución de la capacidad exportadora de las empresas. EI MRLU puede ser expresado matemáticamente como sigue:

$Y_{1 i}^{*}=\beta_{0}+\beta_{1} X_{1}+\beta_{2} X_{2}+\ldots+\beta_{n} X_{n}+u_{i}$

Donde $Y_{1 i}$ es la variable dependiente, explicada o regresando; $X_{1}, X_{2}, \ldots, X_{n}$ son variables explicativas, independientes o regresores; $\beta_{0}$ es la intersección o término "constante"; $\beta_{1}, \beta_{2}, \ldots, \beta_{n}$ son los coeficientes que miden la influencia de $X$ sobre $Y_{1 i}$ y $u_{i}$ es el término de error de la perturbación aleatoria.

Cabe la posibilidad de que los supuestos simplificadores del modelo (Gujarati y Porter, 2010; Wooldridge, 2010) no se ajusten perfectamente a la dinámica de los flujos de exportación; esto obedece a que la decisión empresarial de insertarse en el mercado internacional presenta cierta heterogeneidad interna, por lo que no todos los determinantes de la capacidad exportadora son observables y, en consecuencia, no todas las variables que inciden en la decisión de exportar pueden ser incluidas en el modelo.

Lo anterior implica que si bien la muestra con la que se estima el modelo incluye información contable de todas las empresas santandereanas que son vigiladas por la Superintendencia de Sociedades de Colombia, el método de MCO estima los coeficientes a partir de los reportes de las empresas que vendieron alguna fracción de su producción en el exterior, o lo que es igual, de aquellas que efectivamente exportaron. Este hecho puede generar un sesgo de selección muestral, en razón a que la estimación no contempla la información de la capacidad exportadora de las empresas que no exportan (Heckman, 1979). Dicho de otro modo, si las empresas analizadas no representan a todas las empresas de Santander, es decir, si las empresas que no exportan son excluidas de la estimación, y la capacidad exportadora promedio de las empresas que exportan no refleja la capacidad exportadora que podrían tener las empresas que no exportan si decidieran incursionar en el mercado internacional, 
es posible que la muestra incumpla las condiciones de aleatoriedad (Wooldridge, 2010).

Por este motivo, es conveniente probar si existe sesgo de selección muestral y, en caso afirmativo, corregir la estimación; para tal fin, se utiliza el método de corrección de Heckman (1979). Este procedimiento consta de dos etapas secuenciales. En la primera etapa se utiliza un modelo probit para calcular la probabilidad de que una empresa exporte o no en función de ciertas características internas que se encuentran definidas antes de que esta decida su participación en el mercado exterior $\left(Z_{i}\right)$. En este caso se ha considerado la razón de endeudamiento de la empresa, el retorno operacional sobre los activos (ROA), el tamaño, el sector de actividad económica y su localización en el área metropolitana de Bucaramanga (AMB). Con esta estimación se obtiene un factor de corrección $(\lambda)$ que se utilizará para transformar los coeficientes de la primera estimación por MCO. El modelo probit de selección viene dado por:

$Y_{2 i}{ }^{*}=\alpha_{i} Z_{i}+\varepsilon_{i}$

Donde:

$Y_{2 i}=1$, si $Y_{2 i}{ }^{*}>0$

$Y_{2 i}=0$, si $Y_{2 i}{ }^{*} \leq 0$

$\varepsilon i \sim N(0,1)$

La variable $Y_{2}$ representa la probabilidad de exportar, de modo que cuando se contempla esta probabilidad en $Y_{1}$, la ecuación observada es:

$Y_{1 i}=\beta_{i} X_{i}+u i$, si $Y_{2 i}{ }^{*}>0$

$Y_{1 i}=0$, si $Y_{2 i}{ }^{*} \leq 0$

$\operatorname{Cov}(u i, \varepsilon i)=\rho$

En la segunda etapa se incorpora el factor $\lambda$ (que capta la magnitud del sesgo) como una variable explicativa adicional del modelo y se realiza nuevamente la estimación por MCO. A partir de lo anterior se establece que la ecuación corregida por selección es:

$Y_{1 i}{ }^{*}=\beta_{i} X_{i}+\sigma_{\lambda} \lambda_{i}+u_{i}$

O lo que es igual:

$Y_{1 i}{ }^{*}=\beta_{0}+\beta_{1} X_{1}+\beta_{2} X_{2}+\ldots+\beta_{n} X_{n}+\sigma_{\lambda} \lambda_{i}+u_{i}[5]$
Cuando $\sigma$ (el coeficiente que acompaña a $\lambda$ ) es estadísticamente significativo, se deduce que efectivamente existía un sesgo de selección en la muestra, pero este fue corregido mediante el método de Heckman (1979) en dos etapas. En esas circunstancias se deben interpretar los coeficientes de la nueva estimación. Asimismo, el signo del coeficiente de $\lambda$ indica, en este caso, la relación existente entre decidir vender en el mercado externo y tener capacidad exportadora. Así, si el coeficiente es positivo, la relación es directa; pero si el signo es negativo, entonces la relación es inversa. Por otra parte, si el coeficiente de $\lambda$ no resulta significativo, querrá decir que no existe sesgo de selección muestral, de modo que será necesario analizar los coeficientes obtenidos en la primera estimación del modelo (Heckman, 1979).

\section{Especificación econométrica}

Como se indicó en el apartado anterior, se realiza una estimación econométrica de la relación existente entre la inversión en recursos intangibles y la capacidad exportadora de las empresas santandereanas; dicha capacidad se determina a partir de los flujos de exportación ${ }^{5}$ ponderados por el total de ventas de las empresas. La especificación econométrica del modelo, con sus respectivos controles, viene dada por: $E X P_{i}=\beta_{0}+\beta_{1} P A T_{i}+\beta_{2} M A R_{i}+\beta_{3}$ KNOW $_{i}+$ $\beta_{4} L I C_{i}+\beta_{5} D E R_{i}+\beta_{6} C O N_{-} F R A_{i}+\beta_{7} C R E_{-} M E R_{i}$ $+\beta_{8}$ Endeudamiento $_{i}+\beta_{9} R O A_{i}+\beta_{10} A M B_{i}+$ $\beta_{11}$ Pequeña $_{i}+\beta_{12}$ Mediana $_{i}+\beta_{13}$ Grande $_{i}+$ $\beta_{14}$ Comercio $_{i}+\beta_{15}$ Servicios $_{i}+\beta_{16}$ Construcción $_{i}+$ $\beta_{17}$ Industria $_{i}+\beta_{18}$ Minas $_{i}+u_{i}$

No obstante, con el propósito de verificar la posible existencia de sesgo de selección muestral (y corregirlo en caso afirmativo), se estimará un segundo modelo siguiendo el procedimiento de Heckman (1979). La especificación del modelo corregido está dada como sigue:

$5 \quad$ Los valores de las exportaciones se toman del Directorio de Importadores y Exportadores 2012 de la Dirección de Impuestos y Aduanas Nacionales (DIAN). 
$E X P_{i}=\beta_{0}+\beta_{1} P A T_{i}+\beta_{2} M{ } R_{i}+\beta_{3}$ KNOW $_{i}+$ $\beta_{4} L_{I} C_{i}+\beta_{5} D E R_{i}+\beta_{6} C O N_{-} F R A_{i}+\beta_{7} C R E_{-} M E R_{i}$ $+\beta_{8}$ Endeudamiento $_{i}+\beta_{9} R O A_{i}+\beta_{10} A M B_{i}+$ $\beta_{11}$ Pequeña $_{i}+\beta_{12}$ Mediana $_{i}+\beta_{13}$ Grande $_{i}+$ $\beta_{14}$ Comercio $_{i}+\beta_{15}$ Servicios $_{i}+\beta_{16}$ Construcción $_{i}+$ $\beta_{17}$ Industria $_{i}+\beta_{18}$ Minas $_{i}+\sigma_{\lambda} \lambda_{i}+u_{i}$

A continuación, se listan las variables utilizadas en la estimación de ambos modelos:

- $E X P=$ capacidad exportadora empresarial.

- $\quad P A T=$ millones de pesos COP que la empresa invierte en patentes.

- $M A R=$ millones de pesos COP que la empresa invierte en marcas.

- $K N O W=$ equivalente en millones de pesos COP al valor del know how.

- $\quad$ LIC = millones de pesos COP que la empresa invierte en licencias.

- $D E R=$ millones de pesos COP que la empresa invierte en derechos.

- CON_FRA = millones de pesos COP que la empresa invierte en concesiones y franquicias.

- CRE_MER = millones de pesos COP que la empresa invierte en crédito mercantil.

- Endeudamiento = razón de endeudamiento de la empresa.

- $R O A=$ retorno operacional sobre los activos de la empresa.

- $A M B=$ localización de la empresa en el área metropolitana de Bucaramanga.

- Microempresa (referencia), pequeña, mediana, grande = tamaño de la empresa según sus activos.

- Agropecuario (referencia), comercio, servicios, construcción, industria, minas = sector de actividad económica en el que opera la empresa.

- $\lambda=$ variable de corrección que capta la magnitud del sesgo.

- $u=$ término de error de estimación.

Cabe resaltar que el modelo propuesto sigue la clasificación expresa que hace el Plan Único de Cuentas sobre los recursos que son considerados intangibles en Colombia (Instituto Nacional de Contadores Públicos, 1993): patentes, marcas, know how, licencias, derechos, concesiones y franquicias, y crédito mercantil.

Estos recursos intangibles no se reconocen únicamente como un producto final, pues si bien en algunos momentos las empresas pueden adquirirse, en otras ocasiones conllevan un proceso de creación; por ello, la inversión que registran las empresas contiene tanto los costos de adquisición como de desarrollo. De esta forma, generar estos recursos abarca una gran variedad de habilidades y capacidades estructurales, humanas y relacionales; por lo tanto, los recursos intangibles mencionados reflejan los diferentes tipos de capital intelectual (capital estructural, relacional y humano).

Adicionalmente, para la selección de las variables explicativas se tuvo en cuenta la clasificación de intangibles que plantean autores como Fuentes et al. (2016), López y Nevado (2016), Navas (2015), Núñez y Rodríguez (2014) y GarcíaZambrano, García-Merino y Rodríguez (2014). En estos estudios se resalta que recursos de la organización, tales como derechos comerciales protegidos, derechos de propiedad industrial e intelectual, patentes, know how, marcas, concesiones, franquicias, reputación corporativa, entre otros, tienen la capacidad de generar valor futuro para la empresa, de modo que sobre ellos es posible sustentar una ventaja competitiva sostenida.

\section{Descripción de variables}

En este apartado se describen las variables utilizadas para determinar las implicaciones de la inversión en intangibles sobre la capacidad exportadora de las empresas del departamento de Santander.

\section{Variable dependiente}

Capacidad exportadora empresarial: es la capacidad de penetración de la empresa en los mercados internacionales, medida como la razón entre el valor de las exportaciones y el total facturado por 
concepto de ventas. De acuerdo con lo anterior, cabe resaltar que esta variable toma valores entre 0 y 1 , lo cual indica el porcentaje del total de ventas que se realiza en el mercado exterior.

\section{Variables explicativas}

- Patentes. "Registra el costo de adquisición o de creación y registro de las patentes, las cuales confieren a su titular el derecho a explotar en forma exclusiva la invención por sí mismo, a conceder una o más licencias para su explotación y a percibir regalías o compensaciones derivadas de su explotación por terceros" (Instituto Nacional de Contadores Públicos, 1993, p. 133).

- Marcas. "Registra el costo de adquisición o de producción y registro de signos que, de acuerdo con las normas legales, sirven para distinguir los productos 0 servicios de un ente económico de los de otro. Así mismo registra el costo de marcas catalogadas como colectivas. Se entiende por marca colectiva todo signo calificado de tal, que sirva para distinguir el origen o cualquier otra característica común de productos o de servicios de empresas o colectividades diferentes que utilicen la marca bajo el control del titular" (Instituto Nacional de Contadores Públicos, 1993, p. 133).

- Know how. "Registra el valor apreciable en dinero del conocimiento práctico sobre la manera de hacer o lograr algo con facilidad y eficiencia aprovechando al máximo los esfuerzos, habilidades y experiencias acumuladas en un arte o técnica" (Instituto Nacional de Contadores Públicos, 1993, p. 135).

- Licencias. "Registra el costo o valor pagado al titular de una patente para la explotación de la misma por parte del ente económico" (Instituto Nacional de Contadores Públicos, 1993, p. 136).

- Derechos. "Registra el valor apreciable en dinero que confiere a su dueño el derecho exclusivo de producir y vender material de lectura, grabaciones y obras de arte al amparo de la propiedad intelectual; al igual que aquellos importes incurridos en la adquisición de tales derechos cuando son comprados. Asimismo registra entre otros conceptos, el valor pagado por los puestos en las Bolsas de Valores o Agropecuarias" (Instituto Nacional de Contadores Públicos, 1993, p. 134).

- Concesiones y franquicias. Registra el dinero pagado por la exclusividad concedida por un fabricante o distribuidor para vender sus productos dentro de un territorio específico. También contempla el privilegio concedido por una autoridad gubernamental permitiendo el uso de una propiedad pública que usualmente está sujeto a una regulación especial (Instituto Nacional de Contadores Públicos, 1993, p. 134).

- Crédito mercantil. "Registra el valor adicional pagado en la compra de un ente económico activo, sobre el valor en libros o sobre el valor calculado o convenido de todos los activos netos comprados, por reconocimiento de atributos especiales tales como el buen nombre, personal idóneo, reputación de crédito privilegiado, prestigio por vender mejores productos y servicios y localización favorable. También registra el crédito mercantil formado por el ente económico mediante la estimación de las futuras ganancias en exceso de lo normal, así como de la valorización anticipada de la potencialidad del negocio" (Instituto Nacional de Contadores Públicos, 1993, p. 132). 


\section{ESTIMACIONES Y ANÁLISIS DE RESULTADOS}

En el 2012, las empresas de Santander invirtieron cerca de 374.567 millones de pesos COP en recursos intangibles; de este monto, emplearon el $73,89 \%$ en derechos y el $18,84 \%$ en licencias. Estos tipos de recursos inmateriales son los que registran una mayor inversión en el departamento. De igual forma, el 3,19\% de la inversión se realizó en concesiones y franquicias, el $1,71 \%$ en crédito mercantil, mientras que el valor del conocimiento práctico (know how) tan solo alcanzó el 1,19\%. Las menores inversiones fueron las destinadas para la creación o adquisición de marcas y patentes $(0,92 \%$ y $0,26 \%$, respectivamente). En la tabla 2 se muestra esta distribución.

Tabla 2.

Inversión en recursos intangibles de las empresas de Santander, 2012

\begin{tabular}{|c|c|}
\hline Tipos de recursos intangibles & \% de inversión \\
\hline Derechos & 73,89 \\
\hline Licencias & 18,84 \\
\hline Concesiones y franquicias & 3,19 \\
\hline Crédito mercantil & 1,71 \\
\hline Know how & 1,19 \\
\hline Marcas & 0,92 \\
\hline Patentes & 0,26 \\
\hline
\end{tabular}

Fuente: elaboración de los autores con base en Superintendencia de Sociedades de Colombia (2012).

En este punto es importante resaltar que se han estimado dos regresiones por el método de MCO. En el primer modelo, las variables explicativas corresponden a los siete tipos de recursos intangibles que se detallaron en el apartado anterior, además de algunos controles que capturan características propias de cada empresa. En el segundo modelo se tuvieron en cuenta estas mismas regresoras, pero adicionalmente se incluyó la variable lambda $(\lambda)$ para probar y corregir la posible existencia de un sesgo de selección muestral, mediante el método de corrección de Heckman (1979). Los modelos propuestos fueron estimados en el software econométrico Stata 13 S.E. para el total de empresas de la muestra. Para efectos de cálculo, todas las cifras monetarias fueron expresadas en millones de pesos colombianos y a precios corrientes del 2012.

Las estimaciones de ambos modelos se presentan en la tabla 3. A partir de los resultados, se observa que el coeficiente que acompaña a la variable lambda $(\lambda)$ no es significativo, lo cual indica que los factores que determinan la participación de las empresas en el mercado internacional (razón de endeudamiento, ROA, tamaño, sector y localización) no generan diferencias entre la capacidad exportadora esperada por las empresas no exportadoras y la capacidad exportadora que tienen las empresas exportadoras. Esto sugiere que no existe sesgo de selección y, en consecuencia, se deben analizar los coeficientes estimados del primer modelo (sin corrección de sesgo).

Previo al análisis de los resultados, conviene recordar que el ajuste de una regresión y la validez de los estimadores obtenidos por MCO dependen del cumplimiento de los supuestos básicos del modelo clásico de regresión lineal (Behar, 2003). De este modo, es importante realizar procedimientos conducentes a validar la ausencia de colinealidad entre las variables independientes, la normalidad de los errores y la homocedasticidad de estos (varianza constante a lo largo de las observaciones).

Por lo anterior, con posterioridad a la estimación del modelo, se calculó la matriz de correlaciones entre las variables independientes y se realizó el test de VIF (factor de inflación de la varianza, por sus siglas en inglés) para probar la posible existencia de colinealidad entre las regresoras. Los resultados de estas pruebas dejan en evidencia que no existe multicolinealidad en el modelo (VIF $<10$ para todas las variables explicativas). Asimismo, se realizaron test de Shapiro-Wilk, Shapiro-Francia y de normalidad de curtosis y apuntamiento; los resultados indican que los errores del modelo se distribuyen de forma normal. Finalmente, se realizaron test de White y de Breusch-Pagan para examinar la posible existencia de heterocedasticidad (varianza de los errores no constante). 
Tabla 3.

Resultados de las estimaciones econométricas

\begin{tabular}{|c|c|c|c|c|}
\hline$Y=E X P$ & \multicolumn{2}{|c|}{ Sin corrección de sesgo } & \multicolumn{2}{|c|}{ Con corrección de sesgo } \\
\hline Variable & Coeficiente & Error estándar & Coeficiente & Error estándar \\
\hline PAT & $0,1240^{* * *}$ & 0,0346 & $0,1243^{* * *}$ & 0,0334 \\
\hline MAR & $0,0277^{* * *}$ & 0,0055 & $0,0277 * * *$ & 0,0053 \\
\hline KNOW & $0,0993 * * *$ & 0,0350 & $0,0999 * * *$ & 0,0338 \\
\hline LIC & $0,0181 * *$ & 0,0121 & $0,0179 * *$ & 0,0117 \\
\hline DER & $0,0033^{* *}$ & 0,0026 & $0,0033^{* *}$ & 0,0025 \\
\hline CON_FRA & $0,0268 * * *$ & 0,0098 & $0,0270^{* * *}$ & 0,0095 \\
\hline CRE_MER & $0,0671^{* *}$ & 0,0305 & $0,0672^{* *}$ & 0,0294 \\
\hline Endeudamiento & 0,0126 & 0,0201 & 0,0129 & 0,0194 \\
\hline ROA & 0,0364 & 0,0597 & 0,0370 & 0,0577 \\
\hline AMB & $0,0584 * *$ & 0,0273 & $0,0611^{* *}$ & 0,0271 \\
\hline Microempresa & Referencia & Referencia & Referencia & Referencia \\
\hline Pequeña & $-0,0008$ & 0,0579 & $-0,0005$ & 0,0559 \\
\hline Mediana & $-0,0102$ & 0,0581 & $-0,0105$ & 0,0561 \\
\hline Grande & 0,0174 & 0,0590 & 0,0177 & 0,0569 \\
\hline Agropecuario & Referencia & Referencia & Referencia & Referencia \\
\hline Comercio & $-0,0966^{* * *}$ & 0,0340 & $-0,0964^{* * *}$ & 0,0328 \\
\hline Servicios & $-0,1124^{* * *}$ & 0,0341 & $-0,1120$ *** & 0,0330 \\
\hline Construcción & $-0,0771$ ** & 0,0359 & $-0,0780$ ** & 0,0347 \\
\hline Industria & $-0,0821$ ** & 0,0340 & $-0,0820$ ** & 0,0328 \\
\hline Minas & 0,0060 & 0,0535 & 0,0069 & 0,0517 \\
\hline Constante & 0,0361 & 0,0711 & 0,0331 & 0,0689 \\
\hline$\lambda$ & -- & -- & 0,0877 & 0,2012 \\
\hline Prob > F & \multicolumn{2}{|c|}{0,0000} & \multicolumn{2}{|c|}{--} \\
\hline Prob > chi2 & \multicolumn{2}{|c|}{--} & \multicolumn{2}{|c|}{0,0000} \\
\hline Observaciones & \multicolumn{2}{|c|}{274} & \multicolumn{2}{|c|}{274} \\
\hline
\end{tabular}

* significativo al $10 \%$; ** significativo al $5 \% ;{ }^{* *}$ significativo al $1 \%$.

Prob > F y Prob > chi ${ }^{2}$ corresponden, en cada caso, al p-valor asociado a una prueba de significancia conjunta del modelo, de modo que permite testear la hipótesis nula de que todos los coeficientes del modelo son iguales a cero $(\beta 1=\ldots=\beta 18=0)$. Fuente: elaboración de los autores.

En ambas pruebas, los resultados muestran que los errores son homocedásticos; por consiguiente, se estableció que en el modelo estimado no se violan los supuestos del modelo de regresión lineal y, por tanto, los hallazgos y las conclusiones derivados del análisis de sus resultados son válidos.

En la regresión analizada, los signos de los coeficientes de las variables explicativas son positivos y estadísticamente significativos (tabla 3), lo que confirma la hipótesis de que la inversión en recursos intangibles favorece significativamente la capacidad exportadora de las empresas santandereanas debido a que consolida una ventaja competitiva sostenible, la cual les permite posicionarse en el mercado exterior. Los coeficientes estimados indican que, en Santander, a medida que las empresas incrementan las inversiones en recursos intangibles, su capacidad de penetración en el mercado internacional se incrementa conjuntamente. Estos hallazgos concuerdan con las predicciones teóricas y con la evidencia empírica.

En específico, se encontró que cuando una empresa representativa aumenta en un millón de pesos colombianos (\$1.000.000 COP), el nivel de inversión en patentes (PAT), ceteris paribus, su capacidad exportadora se incrementa cerca del 
$12,4 \%$. Del mismo modo, aumentos equivalentes en los rubros destinados a know how (KNOW) y crédito mercantil (CRE_MER) elevan su potencial de inserción al mercado exterior en 9,9\% y 6,7\%, en su orden. Por su parte, si todas las demás variables organizacionales permanecen constantes, cada millón de pesos COP adicional invertido en marcas (MAR), concesiones y franquicias (CON_FRA), licencias (LIC) y derechos (DER) incide positivamente sobre la capacidad para incursionar en los mercados globales, aunque en menor proporción $(2,7 \%$, $2,6 \%, 1,8 \%$ y $0,3 \%$, respectivamente).

A partir de las estimaciones, se demuestra que la inversión en recursos intangibles es una estrategia financiera que permite el posicionamiento competitivo de las empresas de Santander en el mercado internacional; no obstante, se encontró que existe una relación inversa entre la proporción de la inversión en intangibles y la incidencia en la capacidad exportadora, de modo que los tipos de recursos inmateriales en los que se realizan mayores esfuerzos de inversión son precisamente los que tienen una menor incidencia en la capacidad de penetración al mercado exterior (ver anexo 2).

La explicación de este fenómeno se encuentra en que las implicaciones que tienen los recursos intangibles sobre la capacidad exportadora de las empresas no dependen únicamente de los montos invertidos, sino también de la capacidad y destreza para coordinar dichos recursos. De hecho, autores como Aragón y Rubio (2005) afirman que "la capacidad de la empresa para obtener éxito en los mercados dependerá fundamentalmente de los recursos que posea y de la forma en que sean gestionados" (p. 41). En consecuencia, se puede deducir que en Santander las inversiones en bienes inmateriales no se están gestionando estratégicamente.

\section{CONCLUSIONES Y RECOMENDACIONES}

En la regresión analizada, los signos de los coeficientes de patentes, know how, crédito mercantil, marcas, concesiones y franquicias, licencias y derechos son positivos y estadísticamente significativos; esto indica que a medida que las empresas incrementan las inversiones en recursos inmateriales, su capacidad exportadora se incrementa conjuntamente. Estos hallazgos confirman la hipótesis de que la inversión en intangibles es una estrategia favorable para la inserción de las empresas de Santander en los mercados internacionales. En términos de la teoría de los recursos y capacidades, los intangibles son factores estratégicos o recursos críticos que, si se gestionan estratégicamente, permiten a la empresa lograr una ventaja competitiva sostenida en el tiempo, además de niveles de rentabilidad superiores.

Sin embargo, se encontró una relación inversa entre la proporción de la inversión en intangibles y la incidencia en la capacidad exportadora; de ahí que los tipos de recursos inmateriales en los que se realizan mayores esfuerzos de inversión son precisamente aquellos que tienen una menor incidencia en la capacidad de penetración al mercado exterior. Esta situación implica que, si bien el hecho de invertir en recursos de naturaleza inmaterial es una fuente de ventaja competitiva, las empresas santandereanas que realizan inversiones de este tipo están perdiendo su ventaja comparativa debido a que están invirtiendo en los intangibles menos eficientes en términos de su potencial para incrementar la capacidad exportadora de las empresas.

A partir de los resultados, se hizo un proceso de inducción hacia atrás para tratar de advertir oportunidades estratégicas que permitan encauzar las decisiones de inversión de la comunidad empresarial de Santander. Tras este ejercicio surgieron tres recomendaciones que pueden ser de gran utilidad:

a) Es necesario que las empresas aumenten sus inversiones en recursos intangibles, sobre todo las que se ubican en municipios con menor grado de desarrollo, aquellas de tamaño pequeño y micro, y las que pertenecen a los sectores comercio, servicios, agropecuario y construcción.

b) Es oportuno que las empresas fortalezcan su inversión en los tipos de recursos inmateriales que tienen una mayor incidencia en el incremento de la capacidad exportadora del departamento 
(patentes, know how y crédito mercantil, principalmente).

c) Las organizaciones deben desarrollar actividades de gestión estratégica de sus inversiones en recursos de naturaleza inmaterial.

Puesto que es trascendental que las organizaciones gubernamentales respalden las iniciativas orientadas a fortalecer la ciencia, la tecnología y la innovación en Santander, es importante hacer énfasis en algunas recomendaciones de política pública que pueden incidir positivamente en el desempeño innovador del departamento. En primer lugar, se destaca la necesidad de ejecutar programas que permitan una mayor articulación entre agentes públicos y privados, de manera tal que las estrategias para afianzar la competitividad en el ámbito internacional involucren a los diversos actores del Sistema Regional de Innovación. La segunda recomendación gira en torno al fortalecimiento de los mecanismos de gestión gubernamental que impulsen la creación de mayores sinergias entre microempresas y pequeñas empresas, y fomenten así una cultura asociativa que haga posible incrementar las capacidades para generar y gestionar estratégicamente recursos basados en conocimiento e innovación.

Finalmente, cabe añadir que estas recomendaciones no son la única vía para incrementar la capacidad exportadora; no obstante, su adopción puede brindar una visión más amplia sobre los recursos que otorgan eficiencia organizacional $y$, por tanto, las empresas santandereanas podrán orientar sus esfuerzos al desarrollo de los recursos que tienen mayor potencialidad para brindar una ventaja competitiva sostenida en el tiempo. En consecuencia, esta investigación constituye un estudio pionero en el análisis del alcance que tienen los recursos internos empresariales en la inserción internacional de las empresas del departamento de Santander.

\section{REFERENCIAS}

1. Aragón, A. y Rubio, A. (2005). Factores explicativos del éxito competitivo: el caso de las PyMEs del estado de Veracruz. Revista Contaduría y Administración, 216, 35-69.

2. Barney, J. (1991). Firm resources and sustained competitive advantage. Journal of Management, 17(1), 99-120.

3. Barrios, I. (2005). Modelización del valor intangible y sus determinantes: una aproximación financiera (tesis doctoral). San Cristóbal de La Laguna: Universidad de la Laguna.

4. Behar, R. (2003). Validación de supuestos en el modelo de regresión. Santiago de Cali: Universidad del Valle.

5. Beşkese, A., Bozbura, F. y Aldemir, G. (2014). A model proposal to measure national intellectual capital. Journal of Trends in the Development of Machinery, 18(1), 139-142.

6. Bontis, N. y Mention, A. (2013). Intellectual capital and performance within the banking sector of Luxembourg and Belgium. Journal of Intellectual Capital, 14(2), 286-309.

7. Bontis, N., Janoševic, S. y Dženopoljac, V. (2016). Intellectual capital and financial performance in the Serbian ICT industry. Journal of Intellectual Capital, 17(2), 373-396.

8. Bueno, E. (1998). El capital intangible como clave estratégica en la competencia actual. Boletín de Estudios Económicos, 53(164), 207-229.

9. Bueno, E. (2013). El capital intelectual como sistema generador de emprendimiento e innovación. Revista Economía Industrial, 388, 15-22. 
10. Bueno, E. y Morcillo, P. (1997). Dirección por competencias básicas distintivas: propuesta de un modelo de competitividad y evidencia empírica (Documento de trabajo 51). Madrid: Instituto Universitario de Administración de Empresas de la Universidad Autónoma de Madrid.

11. Capon, N., Farley, J. y Hoenig, S. (1990). Determinants of financial performance: a meta-analysis. Management Science, 36(10), 1143-1159.

12. Catasús, B. y Gröjer, J. (2003). Intangibles and credit decisions: results from an experiment. European Accounting Review, 12(2), 327-355.

13. Congreso de la República de Colombia (1995). Ley 222 de 1995, por la cual se modifica el Libro II del Código de Comercio, se expide un nuevo régimen de procesos concursales y se dictan otras disposiciones. Diario Oficial 42.156.

14. Congreso de la República de Colombia (2006). Decreto 4350 de 2006, por el cual se determinan las personas jurídicas sujetas a la vigilancia de la Superintendencia de Sociedades y se dictan otras disposiciones. Recuperado de https://diario-oficial.vlex.com.co/vid/decreto-354160378

15. Chauvin, K. y Hirschey, M. (1993). Advertising, R\&D expenditures and the market value of the firm. Financial Management, 4, 128-140.

16. Fernández-Jardón, C. y Martos, M. (2014). Capital intelectual y competencias distintivas en pymes madereras de Argentina. Revista de Administração de Empresas, 54(6), 634-646.

17. Fuentes, N., Osorio G. y Mungaray, A. (2016). Capacidades intangibles para la competitividad microempresarial en México. Revista Problemas del Desarrollo, 186(47), 83-106.

18. Fundación COTEC para la Innovación Tecnológica (2012). Tecnología e innovación en España: informe COTEC 2012. Madrid: Autor.

19. Galende, J. y Suárez, I. (1998). Los factores determinantes de las inversiones empresariales en I+D. Economía Industrial, 319, 63-76.

20. García-Rodríguez, F. (2004). La reputación empresarial a partir de la perspectiva basada en los recursos y capacidades: una revisión teórica de su potencialidad competitiva. Investigaciones Europeas de Dirección y Economía de la Empresa, 10(2), 13-27.

21. García-Zambrano, L., García-Merino, J. y Rodríguez, A. (2012). Impacto de la inversión en capital humano sobre el valor empresarial. Revista Latinoamericana de Administración, 51, 15-26.

22. García-Zambrano, L., García-Merino, J. y Rodríguez, A. (2014). Una gestión activa de los intangibles empresariales y su incidencia en los resultados financieros de un modo sostenible. Estudios de Economía Aplicada, 32(3), 1117-1132.

23. Gerencia.com (s. f.). Razones financieras. Recuperado de https://www.gerencie.com/razones-financieras. html.

24. Guerras, L. y Navas, J. (2015). La dirección estratégica de la empresa, teoría y aplicaciones. Madrid: Thomson Reuters-Civitas.

25. Gujarati, D. y Porter, D. (2010). Econometría. Madrid: McGraw-Hill.

26. Gumbau, M. (1997). Análisis microeconómico de los determinantes de la innovación: aplicación a las empresas industriales españolas. Revista Española de Economía, 14(1), 41-66.

27. Heckman, J. (1979). Sample selection bias as a specification error. Econometrica, 47(1), 153-162.

28. Henao, R. y Londoño, A. (2012). Diseño de un modelo de dirección por competencias básicas distintivas para las pymes exportadoras de la ciudad de Medellín. Revista Semestre Económico, 15(32), 197-224. 
29. Huerta, P., Navas, J. y Almodóvar, P. (2004). La diversificación desde la Teoría de Recursos y Capacidades. Cuadernos de Estudios Empresariales, 14, 87-104.

30. Instituto Nacional de Contadores Públicos (1993). Decreto 2650 de 1993, por el cual se modifica el Plan Único de Cuentas para Comerciantes. Recuperado de http://incp.org.co/Site/2012/legislativa/2650.pdf

31. Lev, B. y Sougiannis, T. (1996). The capitalization, amortization and value-relevance of R\&D. Journal of Accounting and Economics, 21, 107-138.

32. López, V. y Nevado, D. (2016). Modelo de control estratégico desde la perspectiva del valor de los intangibles, método y aplicación. Innovar, 26(59), 9-20.

33. Marín, S. (2012). Apropiación social del conocimiento: una nueva dimensión de los archivos. Revista Interamericana de Bibliotecología, 35(1), 55-62.

34. Navas, J. (2015). Reflexiones sobre la identificación y medición del capital intelectual de la empresa. Revista Ciencias Estratégicas, 23(33), 7-13.

35. Núñez, Y. y Rodríguez, C. (2014). Árboles de clasificación para jerarquizar los recursos intangibles asociados a la innovación en las instituciones de educación superior latinoamericanas. Interciencia, 39(3), 149-155.

36. Ordóñez, P. (2004). El capital estructural organizativo como fuente de competitividad empresarial: un estudio de indicadores. Economía Industrial, 357, 131-140.

37. Ostad, K. (1997). Lenders in the information age: financing intellectual property. Secured Lender, 53(6), 134-136.

38. Peluffo, M. y Catalán, E. (2002). Introducción a la gestión del conocimiento y su aplicación al sector público. Santiago de Chile: Cepal.

39. Superintendencia de Sociedades de Colombia (2012). Asuntos económicos y contables. Recuperado de http://www.supersociedades.gov.co/Paginas/default.aspx

40. Superintendencia de Sociedades de Colombia (2017). Quiénes Somos. Recuperado de https://www. supersociedades.gov.co/nuestra_entidad/SitePages/QuienesSomos.aspx

41. Uribe, E. (2001). Los aportes de la dirección por competencias básicas distintivas a la explicación de las operaciones internacionales. Cuadernos de Administración, 14(23), 25-49.

42. Vargas, P. (2003). El impacto de los activos intangibles tecnológicos sobre los resultados empresariales: una aplicación al sector manufacturero español. Recuperado de http://www.madrimasd.org/revista/revista19/ aula/aula1.asp

43. Vickery, G. (1999). La medición de la economía del conocimiento: medición y presentación de los intangibles. Ekonomiaz: Revista Vasca de Economía, 45, 160-187.

44. Villafañe, J. (2005). La gestión de los intangibles empresariales. Comunicação e Sociedade, 8, 101-113.

45. Wernerfelt, B. (1984). A resource-based view of the firm. Strategic Management Journal, 5, 171-180.

46. Wooldridge, J. (2010). Introducción a la econometría: un enfoque moderno. Ciudad de México: Cengage Learning. 


\section{ANEXOS}

\section{Anexo 1. Coeficiente de correlación de Spearman}

En estadística, el coeficiente de correlación de Spearman ( $\rho$ ) es una medida de la correlación (asociación o interdependencia) entre dos variables aleatorias que no se distribuyen normalmente $(X$ y $Y$ ), por lo que toma valores entre -1 y 1 . Para calcular $\rho$, se deben ordenar los pares de datos en función del valor de la variable $X$ y asignarle rangos; luego se debe repetir la ordenación en función de $Y$ y asignarle rangos a esta variable. Para efectos de estimación, el estadístico $\rho$ viene dado por la expresión:

$$
\rho=1-\frac{6 \sum D^{2}}{N\left(N^{2}-1\right)}
$$

Donde:

$N=$ número de pares de datos.

$D=$ diferencia de rangos en las variables de cada par.

Cuando:

$0<\rho<1$ existe una correlación directa o positiva.

$-1<\rho<0$ existe una correlación inversa o negativa.

$\rho=1$ existe una correlación directa perfecta.

$\rho=0$ no existe correlación.

$\rho=-1$ existe una correlación inversa perfecta

Para comprobar la significancia estadística del índice de correlación, se debe probar una hipótesis mediante una aproximación a la distribución $t$ de Student $(\mathrm{T})$, que viene dada por:

$$
T=\frac{\rho}{\sqrt{\frac{\left(1-\rho^{2}\right)}{(N-2)}}}
$$

En este caso:

$\mathrm{H}_{0}: \rho=0 \rightarrow \mathrm{X}$ y $\mathrm{Y}$ no están correlacionadas.

$\mathrm{H}_{\mathrm{a}}: \rho \neq 0 \rightarrow$ existe correlación entre $\mathrm{X}$ y $\mathrm{Y}$.

Para un nivel de confianza del $95 \%$ o superior, se rechazará $\mathrm{H}_{0}$ cuando $\rho \geq t_{\text {critico. }}$. 
Anexo 2. Comparación entre la proporción de inversión en intangibles y la incidencia en la capacidad exportadora de las empresas de Santander

\begin{tabular}{|c|c|c|}
\hline Tipo de recurso intangible & $\begin{array}{c}\text { Incidencia de la inversión en } \\
\text { intangibles sobre la capacidad } \\
\text { exportadora (\%) }\end{array}$ & $\begin{array}{c}\text { Inversión en intangibles de las } \\
\text { empresas de Santander (\%) }\end{array}$ \\
\hline Patentes & 12,4 & 0,2 \\
\hline Know how & 9,9 & 1,1 \\
\hline Crédito mercantil & 6,7 & 1,7 \\
\hline Marcas & 2,7 & 0,9 \\
\hline Concesiones y franquicias & 2,6 & 3,1 \\
\hline Licencias & 1,8 & 18,8 \\
\hline Derechos & 0,3 & 73,8 \\
\hline
\end{tabular}

Fuente: elaboración de los autores. 